\title{
Eosinophile Fasziitis - eine seltene Differenzialdiagnose bei venösen Symptomen
}

\section{Eosinophilic Fasciitis - an uncommon differential diagnosis of venous symptoms}

\author{
Autoren \\ Lena Dietze, Dieter Metze, Tobias Goerge
}

Institut

Hautklinik Uniklinikum Münster

\section{Schlüsselwörter \\ Phlebologie, chronisch venöse Insuffizienz, eosinophile Fasziitis}

Key words

phlebology, chronic venous insufficiency, eosinophilic fasciitis

Bibliografie

Phlebologie 2020; 49: 296-298

DOI 10.1055/a-1171-5047

ISSN 0939-978X

(c) 2020. Thieme. All rights reserved.

Georg Thieme Verlag KG, Rüdigerstraße 14,

70469 Stuttgart, Germany

Korrespondenzadresse

Dr. Lena Dietze

Hautklinik Uniklinikum Münster, Von-Esmarch-Straße 58, 48149 Münster, Deutschland

Lena.Dietze@ukmuenster.de

\section{ZUSAMMENFASSUNG}

Ein 26-jähriger Sportler stellte sich in unserer phlebologischen Ambulanz zur Diagnostik und Therapie bei sichtbarer Varikosis am linken Bein vor. Sonografisch stellte sich eine insuffiziente Vena saphena magna links dar. Es wurde eine VNUSClosure der VSM links komplikationslos durchgeführt. Trotz sonografisch unauffälligen Kontrollen berichtet der Patient im weiteren Verlauf über ein zunehmendes Schweregefühl und eine Verhärtung der Haut. In der Histologie konnte eine eosinophile Fasziitis nachgewiesen werden. Bisher gibt es keine Berichte zum Zusammenhang von chronisch-venöser Insuffizienz, der endoluminalen Behandlung und eosionphiler Fasziitis. Der Fallbericht stellt eine seltene, aber in diesem Fall wichtige Differenzialdiagnose bei postinterventionellen venösen Beschwerden dar.

\section{ABSTRACT}

We present the case of a 26-year-old man who underwent endoluminal treatment of varicose veins of the left leg. Although the operation went successfully without complications the patient presented skin hardening and edema on his extremities about two months later. Histological examination revealed eosinophilic fasciitis. Currently we do not see any correlation between the eosinophilic fasciitis and the endoluminal treatment, the tumescence anaesthetic or varicosis.

\section{Fallbericht}

Ein 26-jähriger Sportler stellte sich in unserer Ambulanz zur phlebologischen Untersuchung bei sichtbarer Varikosis am linken Bein vor. Bis auf einen Nikotinabusus bestanden keine weiteren angiologischen Risikofaktoren, keine Nebenerkrankungen oder die Einnahme einer Regelmedikation.

Duplexsonografisch zeigte sich eine Insuffizienz der Vena saphena magna links IV ${ }^{\circ} \mathrm{n}$. Hach. Es erfolgte die komplikationslose Durchführung einer VNUS-Closure der betroffenen Vene.

In mehrfachen sonografischen Kontrolluntersuchungen zeigte sich ein gutes postinterventionelles Ergebnis. Der Patient berichtete jedoch wiederholt über ein zunehmendes Schweregefühl bei- der Arme und Beine sowie geschwollene Knie beidseits und eine Schwellung des linken Unterarms. Im Verlauf kam es zu einer Einschränkung der Beweglichkeit der Gelenke.

In der körperlichen Untersuchung zeigte sich die Haut der Unterarme verhärtet. Es bestanden Bewegungseinschränkungen im Bereich der Handgelenke und Sprunggelenke.

Zur weiteren Diagnostik entnahmen wir eine tiefe Spindelbiopsie vom Unterschenkel, welche sich vereinbar mit einer eosinophilen Fasziitis zeigte ( $\triangleright$ Abb. 1). Laborchemisch bestand eine Eosinophilie. Wir leiteten eine Therapie mit MTX $15 \mathrm{mg} /$ Woche s. c. sowie flankierend eine Dexamethason-Stoß-Therapie mit jeweils über 3 Tage $100 \mathrm{mg}$ Dexamtheason i. v. alle 4 Wochen für 6 Monate ein. 


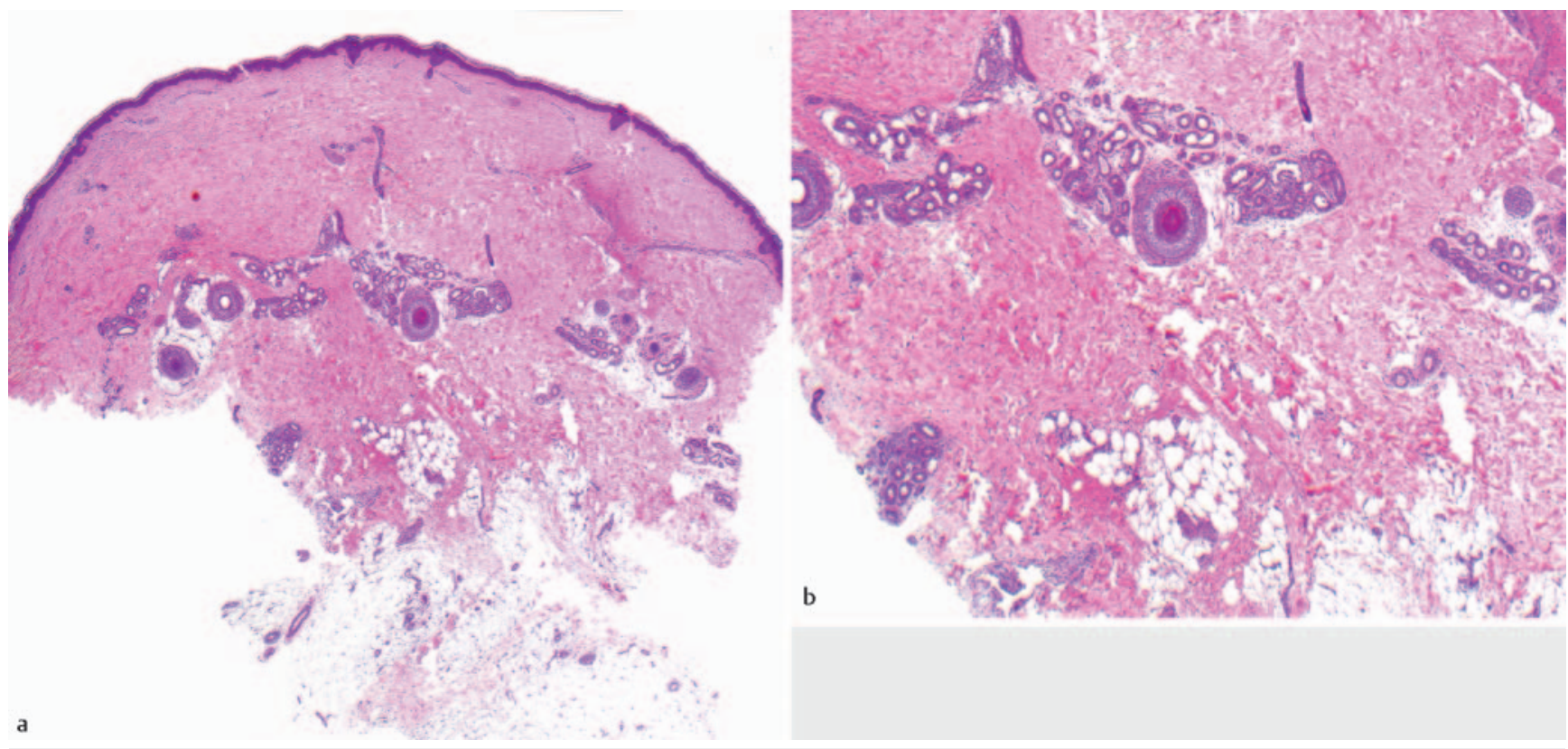

- Abb. 1 Histologischer Befund aus dem Gewebe einer Spindelexzision am Unterarm (Hämatoxylin-Eosin-Färbung). In der Dermis verbreiterte kollagene Fasern sowie bis in die Subkutis perivaskulär sowie periadnexiell diskretes lymphozytäres Infiltrat darstellbar. Es besteht das histologische Bild einer Morphea. Aufgrund entsprechender Klinik und Eosinophilie im Blut ist dieser Befund vereinbar mit einer eosinophilen Fasziitis.

\section{Diskussion}

Die eosinophile Fasziitis ist eine seltene idiopathische Bindegewebserkrankung, bei der es zur Fibrosierung der Haut v. a. an den Extremitäten und am Rumpf kommt [1, 2]. Einige Autoren bezeichnen sie als Unterform der Morphea [2]. Eine autoimmune Genese ist wahrscheinlich [1]. Typischerweise zeigt sich klinisch eine symmetrische Verdickung der Haut der distalen Extremitäten. Es kommt zu einer diffusen Schwellung der Extremitäten mit prall gespannter Haut, die gelegentlich wellige Unebenheiten bildet. Die Haut wird weniger verschieblich und lässt sich im Verlauf nicht mehr abheben. Als typisches klinisches Zeichen wird das negative Venenzeichen beschrieben [3]. Hierbei kommt es zur strichförmigen Eindellung statt zum typischen Hervortreten der großen Hautvenen und aufgrund des erhöhten Drucks der verhärteten Haut kollabieren die Venen [3]. Im Verlauf kann es zur dauerhaften dermatogenen Kontraktur von Gelenken kommen, wenn diese aufgrund der Hautverhärtung eingeschränkt bewegbar sind [1].

Laborchemisch lässt sich häufig eine Eosinophilie nachweisen, welche jedoch keine Korrelation zur Krankheitsaktivität zeigt [1, 4]. Zur histologischen Sicherung ist die Entnahme einer tiefen Spindelbiopsie nötig, welche Faszie und Muskelgewebe einschließt [2]. Histologisch sind entzündliche Infiltrate bis zu den Faszien mit Lymphozyten, Makrophagen und Eosinophilen nachweisbar [5]. Dermis und Epidermis können unauffällig oder ebenfalls fibrosiert sein $[1,5]$.

Zur Diagnosestellung wurden 2014 von Pinal-Fernandez et al. als Hauptdiagnosekriterien eine Verdickung der Haut in Verbindung mit histologisch nachweisbaren Entzündungsinfiltraten vorgeschlagen.
Zur Therapie der eosinophilen Fasziitis werden systemische Glukokortikoide als First-line-Therapie eingesetzt. Second-line sind Therapien mit Methotrexat in niedriger Dosierung (15$25 \mathrm{mg} /$ Woche) [1, 4]. Spontanremissionen der eosinophilen Fasziitis sind beschrieben [6].

Die Ätiologie und der Pathomechanismus der Erkrankung sind ungeklärt. Es sind Fälle beschrieben, in denen ein Zusammenhang mit anstrengendem körperlichem Training nachgewiesen werden konnte [7, 8]. Des Weiteren wurden Patienten mit posttraumatischer eosinophiler Fasziitis beschrieben [9, 10]. Es gibt aktuell jedoch keine Hinweise darauf, dass ein kausaler Zusammenhang zwischen dem Auftreten einer eosinophilen Fasziitis im Rahmen einer chronisch-venösen Insuffizienz oder deren Behandlung mit einer endoluminalen Behandlung besteht. Auch eine Assoziation der Tumeszenz-Anästhesie mit dem Auftreten einer eosinophilen Fasziitis ist uns nicht bekannt, sodass wir aktuell davon ausgehen, dass es sich um 2 unabhängige Krankheitsgeschehen handelt. Die eosinophile Fasziitis stellt eine wichtige Differenzialdiagnose bei Symptomen wie Erythem, Schwellung und Ödem dar. Sollte es zu oben beschriebener Symptomatik kommen, ist eine histologische Untersuchung sinnvoll. Glücklicherweise konnte die eosinophile Fasziitis im Rahmen der phlebologischen Nachkontrollen diagnostiziert werden und eine rechtzeitige Behandlung eingeleitet werden.

Interessenkonflikt

Die Autorinnen/Autoren geben an, dass kein Interessenkonflikt besteht. 


\section{Literatur}

[1] Pinal-Fernandez I, Selva-O'Callaghan A, Grau JM. Diagnosis and classification of eosinophilic fasciitis. Autoimmunity reviews 2014; 13 (4): 379-382

[2] Mertens JS, Seyger MM, Thurlings RM et al. Morphea and eosinophilic fasciitis: an update. American Journal of Clinical Dermatology 2017; 18 (4): 491-512

[3] Fruchter R, Mazori DR, Femia AN. Groove sign of eosinophilic fasciitis. JCR: Journal of Clinical Rheumatology 2017; 23 (3): 169

[4] Lebeaux D, Francès C, Barete $S$ et al. Eosinophilic fasciitis (Shulman disease): new insights into the therapeutic management from a series of 34 patients. Rheumatology 2012; 51 (3): 557-561

[5] Toquet C, Hamidou MA, Renaudin K et al. In situ immunophenotype of the inflammatory infiltrate in eosinophilic fasciitis. The Journal of rheumatology 2003; 30 (8): 1811-1815
[6] Michet JC, Doyle JA, Ginsburg WW. Eosinophilic fasciitis: report of 15 cases. In Mayo Clinic Proceedings 1981; 56 (1): 27-34

[7] Sturdy A, Stratton R, Perez-Machado M et al. Case of eosinophilic fasciitis during military training in a Nepalese British infantry soldier. BMJ Mil Health

[8] Ihn H. (2019). Eosinophilic fasciitis: From pathophysiology to treatment. Allergology International 2020; 68 (4): 437-439

[9] Romero AG, Fernandez JG, Calatayud JC. Eosinophilic fasciitis associated with simple traumatism. Acta dermatovenerologica Croatica: ADC 2001; 9 (4): 287

[10] Antic M, Lautenschlager S, Itin PH. Eosinophilic fasciitis 30 years after what do we really know? Dermatology 2006; 213 (2): 93-101 